\title{
Partial duplication of the CRYBB1-CRYBA4 locus is associated with autosomal dominant congenital cataract
}

\author{
Owen M Siggs ${ }^{\star, 1,5}$, Shari Javadiyan ${ }^{1,5}$, Shiwani Sharma ${ }^{1}$, Emmanuelle Souzeau ${ }^{1}$, Karen M Lower ${ }^{2}$, \\ Deepa A Taranath ${ }^{1}$, Jo Black ${ }^{3}$, John Pater ${ }^{1,3}$, John G Willoughby ${ }^{1}$, Kathryn P Burdon ${ }^{\star, 4}$ and Jamie E Craig ${ }^{1,6}$ \\ Congenital cataract is a rare but severe paediatric visual impediment, often caused by variants in one of several crystallin genes \\ that produce the bulk of structural proteins in the lens. Here we describe a pedigree with autosomal dominant isolated \\ congenital cataract and linkage to the crystallin gene cluster on chromosome 22. No rare single nucleotide variants or short \\ indels were identified by exome sequencing, yet copy number variant analysis revealed a duplication spanning both $C R Y B B 1$ and \\ CRYBA4. While the CRYBA4 duplication was complete, the CRYBB1 duplication was not, with the duplicated CRYBB1 product \\ predicted to create a gain of function allele. This association suggests a new genetic mechanism for the development of isolated \\ congenital cataract.
}

European Journal of Human Genetics (2017) 25, 711-718; doi:10.1038/ejhg.2017.33; published online 8 March 2017

\section{INTRODUCTION}

Cataract is an opacification of the crystalline lens, and one of the leading causes of blindness worldwide. ${ }^{1}$ Those that occur within the first year of life are categorised as congenital or infantile cataract, with an incidence in the order of 52.8 per 100000 children. $^{2}$ Around $23 \%$ of non-syndromic congenital cataracts are familial, ${ }^{3}$ with around $50 \%$ of these associated with a crystallin gene variant. ${ }^{4}$

Crystallin proteins account for more than $90 \%$ of soluble lens protein, and can be divided into $\alpha$-, $\beta$ - and $\gamma$-crystallin families. With age, the closely related $\beta$ - and $\gamma$-crystallins gradually form insoluble aggregates, which the chaperone-like $\alpha$-crystallins serve to counteract. Around 10 human crystallin genes are known to be mutated in congenital cataract, with variants typically thought to reduce crystallin solubility directly (eg, by creating an insoluble $\beta$ - or $\gamma$-crystallin) or indirectly (eg, due to loss of $\alpha$-crystallin chaperone function). ${ }^{5}$ These genes include both $\alpha$-crystallins (CRYAA and CRYAB), two acidic $\beta$-crystallins (CRYBA1 and CRYBA4), three basic $\beta$-crystallins (CRYBB1, CRYBB2, and CRYBB3), and three $\boldsymbol{\gamma}$-crystallins (CRYGC, CRYGD, and CRYGS). ${ }^{4}$

The chromosomal arrangement of human crystallin genes reflects their evolutionary history, with major clusters on chromosomes 2 and $22 .{ }^{6}$ Of a total of eight $\gamma$-crystallin genes, six are located on chromosome 2. Similarly, all three basic $\beta$-crystallin genes (CRYBB1, $C R Y B B 2$, and CRYBB3) are located on chromosome 22, with the acidic $\beta$-crystallin $C R Y B A 4$ directly adjacent to $C R Y B B 1$ (but transcribed in the opposite direction). This CRYBB1-CRYBA4 arrangement is present in organisms as distant as zebrafish, and likely significant for their coordinate regulation. Either gene can lead to congenital cataract when mutated: CRYBA4 missense variants are known to cause dominant cataract, ${ }^{7}$ while $C R Y B B 1$ variants may be dominant ${ }^{8}$ or recessive. ${ }^{9}$

Here we describe an autosomal dominant congenital cataract pedigree associated with a unique duplication of the paired CRYBB1-CRYBA4 locus. Both genes were found to be duplicated, with a complete duplication of CRYBA4 and partial duplication of CRYBB1.

\section{MATERIALS AND METHODS}

Patients

Clinical information from 19 members of pedigree CSA106 (Caucasian) was collected by referring ophthalmologists (Table 1), with blood samples taken after informed written consent. Of these, 11 had developed bilateral cataracts. The study was approved by the Southern Adelaide Clinical Human Research Ethics Committee.

\section{Candidate gene panel screening}

Individual CSA106.03 (Figure 1a) was screened for variants in 51 known congenital cataract genes using an Ion AmpliSeq custom amplicon panel (Life Technologies, Carlsbad, CA, USA). Genomic DNA concentration was measured with a dsDNA high sensitivity Assay Kit on a Qubit fluorometer (Life Technologies). Library preparation (Ion AmpliSeq library kit v2.0) and template preparation (Ion PGM Template OT2 200 Kit) were performed according to the manufacturer's instructions. The clonally amplified library was enriched on the Ion OneTouch enrichment system and quantified with a Bioanalyzer 2100 using the High Sensitivity DNA Kit (Agilent Technologies, Santa Clara,

\footnotetext{
${ }^{1}$ Department of Ophthalmology, Flinders University, Flinders Medical Centre, Bedford Park, South Australia, Australia; ${ }^{2}$ Department of Haematology and Genetic Pathology, Flinders University, Bedford Park, South Australia, Australia; ${ }^{3}$ Department of Ophthalmology, Women's and Children's Hospital, North Adelaide, South Australia, Australia; ${ }^{4}$ Menzies Institute for Medical Research, University of Tasmania, Hobart, Tasmania, Australia

${ }^{*}$ Correspondence: Dr OM Siggs, Department of Ophthalmology, Flinders University, Flinders Medical Centre, 1 Flinders Drive, Bedford Park South Australia 5042, Australia. Tel: +61 88204 4899; Fax: +61 88204 6722; E-mail: sigg0005@flinders.edu.au

or Associate Professor KP Burdon, Menzies Institute for Medical Research, University of Tasmania, 17 Liverpool St (Private Bag 23), Hobart, Tasmania 7000, Australia. Tel: +61 36226 4288; Fax: +61 36226 7704; E-mail: Kathryn.burdon@utas.edu.au

5 Joint first author.

${ }^{6}$ Joint senior author.

Received 26 February 2016; revised 22 January 2017; accepted 1 February 2017; published online 8 March 2017
} 
Table 1 Clinical details of CSA106 family members

\begin{tabular}{|c|c|c|c|c|c|c|c|}
\hline ID & Age diagnosed (y) & Exome sequenced & Disease Status & Age of surgery (y) (RE) & Age of surgery $(y)(L E)$ & pre-surgical VA (RE) & pre-surgical VA (LE) \\
\hline CSA106.02 & 0 & Yes & Affected & 44 & 44 & NA & NA \\
\hline CSA106.04 & 3 & Yes & Affected & 18 & 19 & NA & NA \\
\hline CSA106.05 & - & Yes & Unaffected & - & - & - & - \\
\hline CSA106.06 & 4 & No & Affected & 7 & 13 & $6 / 36$ & $6 / 12$ \\
\hline CSA106.09 & - & Yes & Unaffected & - & - & - & - \\
\hline CSA106.10 & - & No & Unaffected & - & - & - & - \\
\hline CSA106.11 & - & No & Unaffected & - & - & - & - \\
\hline CSA106.12 & NA & No & Affected & NA & NA & NA & NA \\
\hline CSA106.13 & NA & No & Affected & NA & NA & NA & NA \\
\hline CSA106.14 & 10 & Yes & Affected & 51 & 10 & NA & NA \\
\hline CSA106.19 & NA & Yes & Affected & NA & NA & NA & NA \\
\hline
\end{tabular}

Abbreviations: LE, left eye; NA, not available; RE, right eye; VA, visual acuity; y, years. All affected members had bilateral cataracts.

CA, USA). Sequencing was performed on an Ion Torrent Personal Genome Machine (PGM) using The Ion PGM Sequencing 200 Kit v2 and an Ion 318 chip (Life Technologies). Torrent Suite (v3.6) was used to align reads to the hg19 reference genome. The number of mapped reads, percentage of ontarget reads, and mean read depth were calculated using the Coverage Analysis plugin (v4.0-r77897), and variants were called using the Variant Caller plugin (v4.0-r76860) with germline algorithm. Ion Reporter v4.0 was used for annotation. Variants were prioritised for further analysis if they were predicted to alter protein coding sense, were rare (mean allele frequency $<0.001$ in Exome Variant Server (http://evs.gs.washington.edu/ $\mathrm{EVS} /$ )), and were absent from unaffected controls.

\section{Exome sequencing}

A total of 11 members of the CSA106 family (six affected, five unaffected) were subjected to exome sequencing, along with an additional 325 unrelated Australian cases and controls (a mixture of examined normal controls (20), cataract cases (22), keratoconus cases (51), advanced glaucoma cases (195), and primary congenital glaucoma cases (37)). Genomic DNA was extracted from blood samples using a QIAamp Blood Maxi Kit (Qiagen, Hilden, Germany) and subjected to exome capture (Agilent SureSelect v4). Paired-end libraries were then sequenced on an Illumina HiSeq 2000 by an external contractor (Axeq Technologies, Rockville, MD, USA). Reads were mapped to the human reference genome (hg19) using the Burrows-Wheeler Aligner (v0.7.10), and duplicates were marked and removed using Picard (v1.126). An average of 49 429126 reads per sample were mapped to capture regions, with a mean read depth of 83.6 and $>\times 10$ coverage for $97.3 \%$ of the capture regions. Variants were called using SAMtools (v1.0) and annotated against RefSeq transcripts using ANNOVAR (2014Nov12) with additional annotations from the NHLBI Exome Sequencing Project (ESP6500SI-V2), 1000 Genomes Project (November 2014 release), ExAC (v0.3.1), and dbSNP138 databases. Variants were filtered by QUAL score $(>20)$, and considered to be potentially pathogenic if predicted to alter protein coding sense (nonsynonymous, stopgain, stoploss, frameshift, essential splice), and were sufficiently rare (ExAC mean allele frequency $<0.0001)$. CRYBB1 variants were annotated according to RefSeq accession numbers NG_009826.1, NM_001887.3 and NP_001878.1, with CRYBA4 variants based on NG_009825.1, NM_001886.2 and NP_001877.1.

\section{Linkage analysis}

VCF files were converted to MERLIN input format using the vcf2linkdatagen and linkdatagen scripts. ${ }^{10}$ Parametric linkage analysis was then performed using
MERLIN (v1.1.2) under a fully penetrant dominant model with a disease frequency of 0.0001 .

\section{Exome CNV analysis}

Coverage depth across the critical region was extracted from exome BAM files using SAMtools (v1.3). For copy number variant analysis using CoNIFER (v0.2.2), the same interval was analysed in 343 population-matched control exomes (including 11 from family CSA106) using the following parameters: SVD 5, ZRPKM 1.5.

\section{qPCR CNV analysis}

TaqMan Copy Number Assays for intron 3 (Hs04088405_cn: hg19 chr22: g.27006444) and exon 6 (Hs00054226_cn: hg19 chr22:g.26995522) of CRYBB1 were ordered from ThermoFisher Scientific. All available CSA106 family members were tested for partial duplication in CRYBB1 gene using genomic DNA according to manufacturer's protocol. Briefly, each region was amplified in four replicates on a StepOne Plus real-time PCR instrument alongside an endogenous reference gene (TaqMan Copy Number Reference Assay, human, RNase P). CopyCaller 2.0 software (Life Technologies) was used to predict the copy number of the target genomic DNA. Intron 3 was also screened in an additional 46 congenital cataract probands with an unidentified genetic cause (recruited under the same protocol as the CSA106 family).

\section{Whole-genome sequencing}

Genomic DNA from a single affected family member (CSA106.19) was extracted from a blood sample using a QIAamp Blood Maxi Kit (Qiagen). A 150 bp paired-end library was generated using the TruSeq Nano kit (v2.5) and sequenced on an Illumina HiSeq X Ten system by an external contractor (Garvan Institute of Medical Research, Darlinghurst, NSW, Australia). Sequence data of $148.75 \mathrm{~Gb}$ was generated, with reads mapped to the human reference genome (hg19) using the Burrows-Wheeler Aligner (v0.7.12), and duplicates marked and removed using Picard (v2.6). Approximately 988463168 reads were mapped to the hg19 reference genome, with a mean read depth of 43.3 and $>\times 10$ coverage for $96.8 \%$ of the genome. Local realignment and base quality recalibration was performed using the Genome Analysis Toolkit (GATK, v3.5). The predicted pathogenic variant identified in this study has been submitted to the ClinVar Database (accession number SCV000484507, https://www.ncbi.nlm.nih.gov/clinvar/). 
a

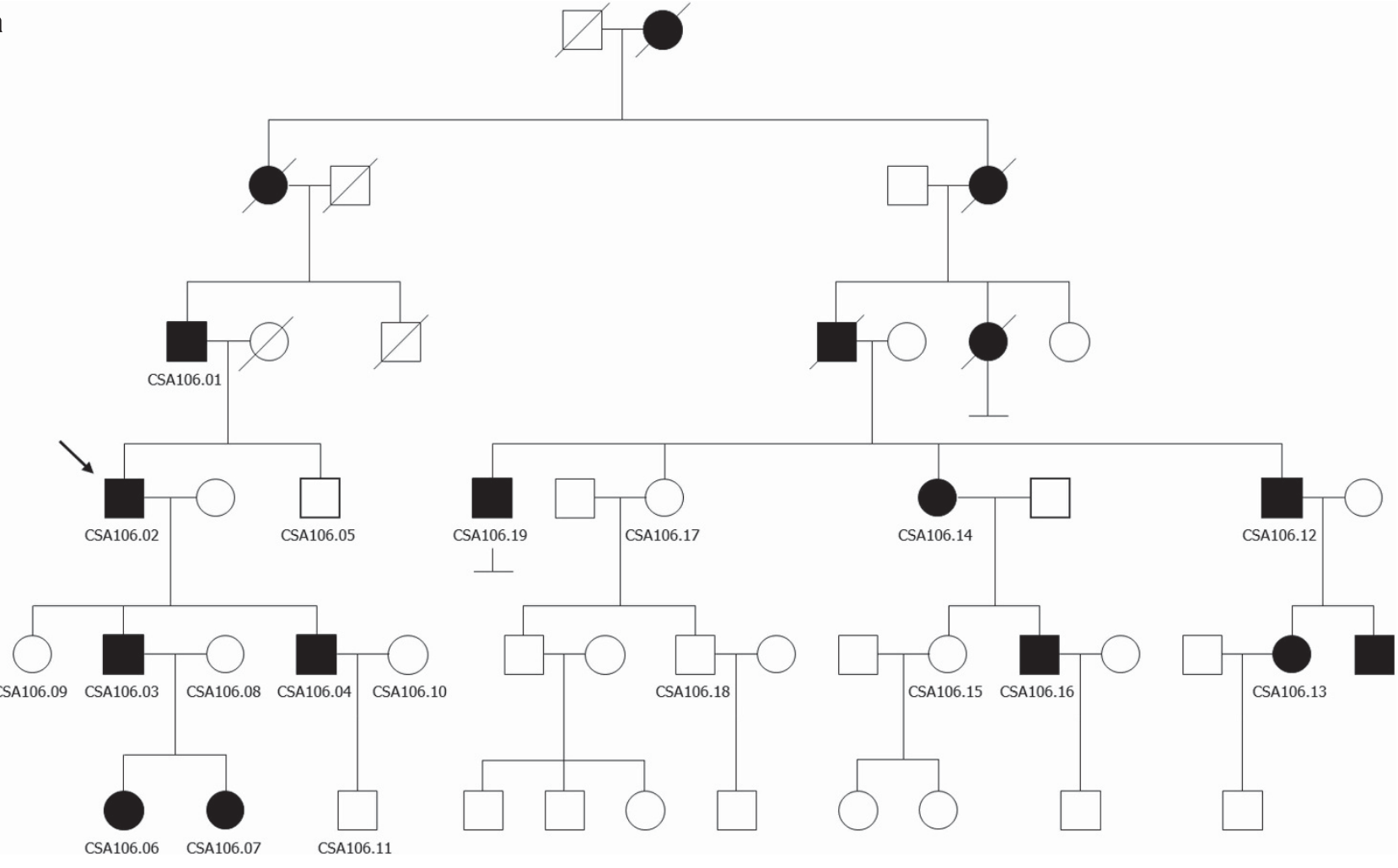

b

$$
\text { CSA106.02 (LE) }
$$
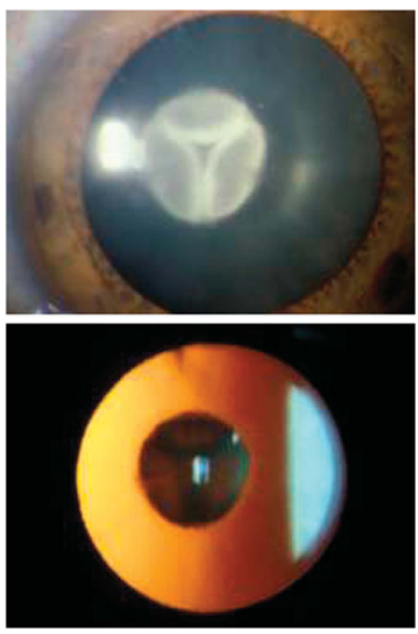

CSA106.04 (RE)
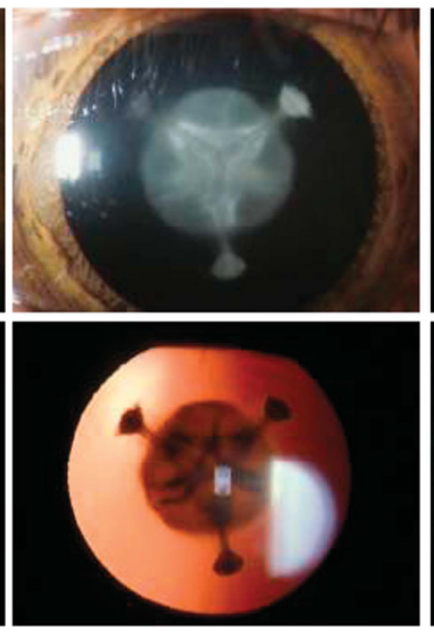

CSA106.06 (LE)
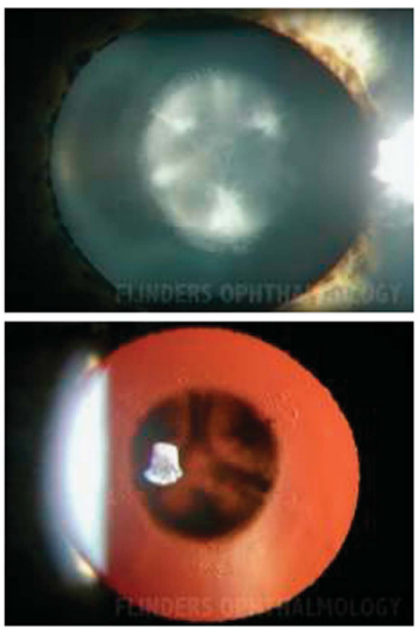

CSA106.07 (LE)
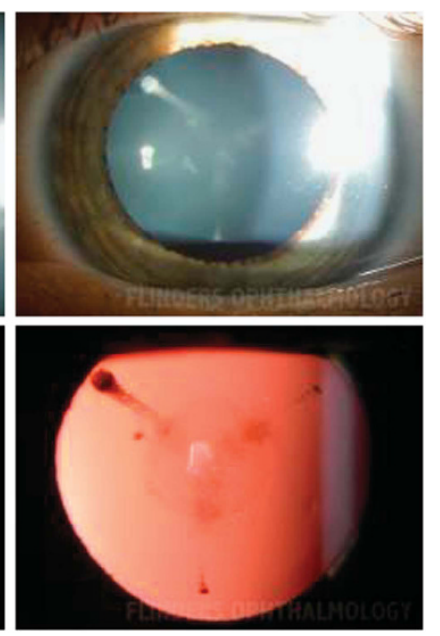

Figure 1 An autosomal dominant congenital cataract pedigree. (a) CSA106 pedigree, indicating affected (black), and unaffected (white) members. Proband (CSA106.02) is indicated by an arrow. (b) Direct illumination (top panels) or retroillumination (bottom panels) of the same cataract from four family members, demonstrating mild to dense fetal nuclear opacification with sutural involvement. LE, left eye; RE, right eye.

\section{Lens protein extraction}

Cataractous lens material was collected from the proband during phacoemulsification (CSA106.6, aged 13 years), and stored in balanced salt solution with 2 mM EDTA pH 8.0 at $-80^{\circ} \mathrm{C}$. Normal human lens was obtained from an 18 year-old deceased donor (Eye Bank of South Australia, Flinders Medical Centre), collection of which was approved by Southern Adelaide Clinical Human Research Ethics Committee. Lenses were homogenised in $2 \mathrm{ml}$ of extraction buffer containing $50 \mathrm{~mm}$ imidazole ( $\mathrm{pH} 7), 50 \mathrm{~mm} \mathrm{NaCl}, 2 \mathrm{~mm}$ 6-aminohexanoic acid, $1 \mathrm{~mm}$ EDTA, and protease inhibitor cocktail (Roche Diagnostics, Basel, Switzerland), and ultracentrifuged at $150000 \mathrm{~g}$ for $30 \mathrm{~min}$ at $4{ }^{\circ} \mathrm{C}$. The soluble fraction was acetone precipitated according to the Thermos
Scientific protocol (Thermo Fisher, Waltham, MA, USA). The insoluble fraction was dissolved in buffer containing $6 \mathrm{M}$ urea, $2 \%$ dichloro-diphenyltrichloroethane, 2\% 3-[C3-cholamidoproyl] dimethyl-ammonio-1propansulfonat and $0.1 \%$ sodium dodecyl sulfate (SDS). The EZQ Protein Quantitation method (Life Technologies) was used to determine protein concentration.

\section{Denaturing gel electrophoresis and western blotting}

Around $20 \mu \mathrm{g}$ of total soluble protein from each lens was size fractionated by SDS-PAGE using a $12 \%$ polyacrylamide gel. The precision plus protein standards (BioRad, Hercules, CA, USA) were used for size comparison. 
Fractionated proteins were transferred onto Hybond-C Extra nitrocellulose (GE Healthcare, Little Chalfont, UK), and after blocking in 5\% (wt/vol) milk in TBS-Tween (Tris Buffered Saline and 1\% Tween-20) was incubated with a mouse monoclonal anti-CRYBB1 primary antibody (1:400, Sigma-Aldrich, St Louis, MO, USA; WH0001414M3). After washing the membrane was incubated with horseradish peroxidase (HRP) -conjugated goat anti-mouse IgG (1:1000, Jackson ImmunoResearch, West Grove, PA, USA), and following another wash was treated with Clarity Western ECL Blotting Substrate (BioRad) or Amersham ECL Prime (GE Healthcare) and imaged using an ImageQuant LAS 4000 Imager (GE Healthcare). The same membrane was stripped at $50^{\circ} \mathrm{C}$ in stripping buffer (100 mM $\beta$-mercaptoethanol, $2 \%$ SDS and $62.5 \mathrm{~mm}$ Tris$\mathrm{HCl}[\mathrm{pH} 7])$ then reprobed with polyclonal rabbit anti-CRYBA4 (1:200, Abcam, Cambridge, UK; ab130680) or polyclonal sheep anti-CRYAA (1:1000; Flinders University Antibody Production Facility) primary antibodies, followed by HRP-conjugated goat anti-rabbit IgG or anti-sheep IgG secondary antibodies (1:1000, Jackson ImmunoResearch).

\section{RESULTS}

\section{Autosomal dominant congenital cataract}

We identified a six-generation autosomal dominant congenital cataract pedigree (Figure 1a), with multiple affected members diagnosed with nonsyndromic bilateral cataracts between birth and 10 years of age (Table 1). Slit lamp imaging of the lens demonstrated mild to dense fetal nuclear opacification with anterior and posterior sutural involvement (Figure 1b). A custom amplicon sequencing panel, designed to sequence the exons of 51 known congenital cataract genes (Supplementary Table 1), was first used to screen an affected family member (CSA106.03). A total of 807055 reads were mapped to the reference genome, covering $93.21 \%$ of target gene bases to at least $\times 20$ depth (average read depth of 623.5 across 1216 amplicons). A total of 172 variants were identified across the 51-gene panel: all of which either had a mean allele frequency of greater than 0.001 , were not predicted to alter protein sequence, or were present in concurrently sequenced control samples.

\section{Linkage to chromosome 22}

Given the absence of a candidate variant in a known congenital cataract gene that affects function, we sequenced the exomes of 11 family members (six affected, five unaffected). Parametric linkage analysis under a rare dominant inheritance model revealed a peak LOD score of 3.3 on chromosome 22 (Figure 2a and b). Haplotype phasing indicated that rs2236005 (hg19 chr22:g.26422980A $>$ G) and rs2347790 (hg19 chr22:g.29414001A > G) were the boundaries of the 3 Mbp critical region (Figure 2c), within which lay 17 protein-coding genes (plus 9 ncRNA and 7 pseudogenes) including the known congenital cataract genes CRYBB1 and CRYBA4 (Figure 2d). All 17 protein-coding gene exons were covered by exome capture sequencing, with CRYBB1 and CRYBA4 also having been previously covered by candidate gene amplicon sequencing. We detected two singlenucleotide variants that were shared between affected family members, absent from unaffected family members, and that altered coding sense (Figure 2e). Both variants had a mean allele frequency at least an order of magnitude greater than the estimated population incidence of congenital cataract $(\sim 0.000528)$, and therefore were considered extremely unlikely candidates. Synonymous variants in CRYBB1 or CRYBA4-recently identified as a possible cause of crystallin misfolding ${ }^{11}$-were also not shared between affected members.

\section{Partial duplication of the CRYBB1-CRYBA4 locus}

We next investigated coverage depth across the linkage interval using two methods. Both CoNIFER (Figure 3a) and SAMtools (Figure 3b) revealed an increased coverage depth (and by inference increased copy number) at the CRYBB1-CRYBA4 locus of all affected individuals. Based on coverage of protein-coding exons across the locus, this CNV spanned between a minimum of $28.8 \mathrm{kbp}$ (hg19 chr22:g.26997843_ 27026636) and a maximum of $1.15 \mathrm{Mbp}$ (hg19 chr22:g.26995638_ 28146902), the smaller of which encompassed only two proteincoding genes (CRYBB1 and CRYBA4), and the larger of which also included a long non-coding RNA gene (MIAT). This CNV (hg19 chr22:g.(26995638_26997843)_(27026636_28146902)dup) was present in all six affected family members, absent from all five unaffected family members, and was absent from a further 325 unrelated Australian exomes sequenced contemporaneously. Mean coverage depth analysis revealed that while all five captured exons of CRYBA4 appeared to be duplicated (representing the final five of six total exons), only the first five exons of CRYBB1 (of a total of six) appeared to have been duplicated (Figure $3 \mathrm{~b}$ ). Duplication of CRYBB1 intron 3 was confirmed by qPCR in all affected family members, with a similar assay showing that distal exon 6 was not duplicated (Figure $3 \mathrm{c}$ and d). CRYBB1 and CRYBA4 variation was also manually inspected via Integrative Genomics Viewer, given that a variant in one CRYBB1-CRYBA4 locus of a total of three (two alleles plus the duplicated allele) may not be called as heterozygous by variant-calling algorithms. We also screened an additional 46 unsolved congenital cataract cases for CRYBB1 duplications, yet did not identify any further duplications (Figure 3e).

To examine the effects of the CRYBB1-CRYBA4 duplication on protein expression, we prepared protein from the cataractous left lens of CSA106.06 (removed during phacoemulsifcation) and from a noncataractous control lens. An anti-CRYAA Western blot indicated equivalent loading between the cataract and control samples, and reprobing the same blot with anti-CRYBA4 and anti-CRYBB1 blots revealed bands of the appropriate size $(22 \mathrm{kDa}$ and $28 \mathrm{kDa}$ monomers, respectively) and similar density in both samples (Figure 3f). An additional band was detected with the anti-CRYBA4 antibody corresponding to a CRYBA4 dimer ( $44 \mathrm{kDa})$, although no other bands were apparent. We did not detect any additional anti-CRYBB1-reactive bands in the cataract sample in soluble fractions (Figure 3f), or insoluble fractions (data not shown), despite using an antibody raised against a peptide (NP_001878, p.37_138) that is not encoded by exon 6 .

\section{Tandem duplication of the CRYBB1-CRYBA4 locus}

To define the nature of the duplication and the associated breakpoints, we performed whole-genome sequencing on genomic DNA from an affected family member (CSA106.19). Read depth analysis confirmed the presence of a 78,928 bp duplication at the CRYBB1-CRYBA4 locus (hg19 chr22:g.26995597_27074524dup), the boundaries of which occurred immediately proximal to chr22:26995597, and distal to chr22:27074524 (Figure 4a). This duplication encompassed the entirety of CRYBA4 and the long non-coding RNA gene MIAT, but only partially involved $C R Y B B 1$. Importantly, the proximal duplication boundary occurred within exon 6 of CRYBB1, just 75 bp distal to the site of the exon $6 \mathrm{qPCR}$ probe used in Figure 3d. This also explains why a duplicated exon 6 was not detected by exome sequencing, as there would be insufficient complementarity to the exon 6 capture probe.

The arrangement of multiple discordant mate pairs within the duplicated region (Figure 4a) suggested a direct tandem duplication. ${ }^{12}$ This was confirmed by BLAST alignments of split reads spanning the duplication breakpoint, which revealed a proximal breakpoint joining exon 6 of CRYBB1 to intergenic sequence distal to MIAT (Figure 4b). No insertions, deletions, or other rearrangements were evident at either proximal or distal breakpoints, although there was $3 \mathrm{bp}$ of microhomology at the adjoining sequences of the proximal breakpoint 


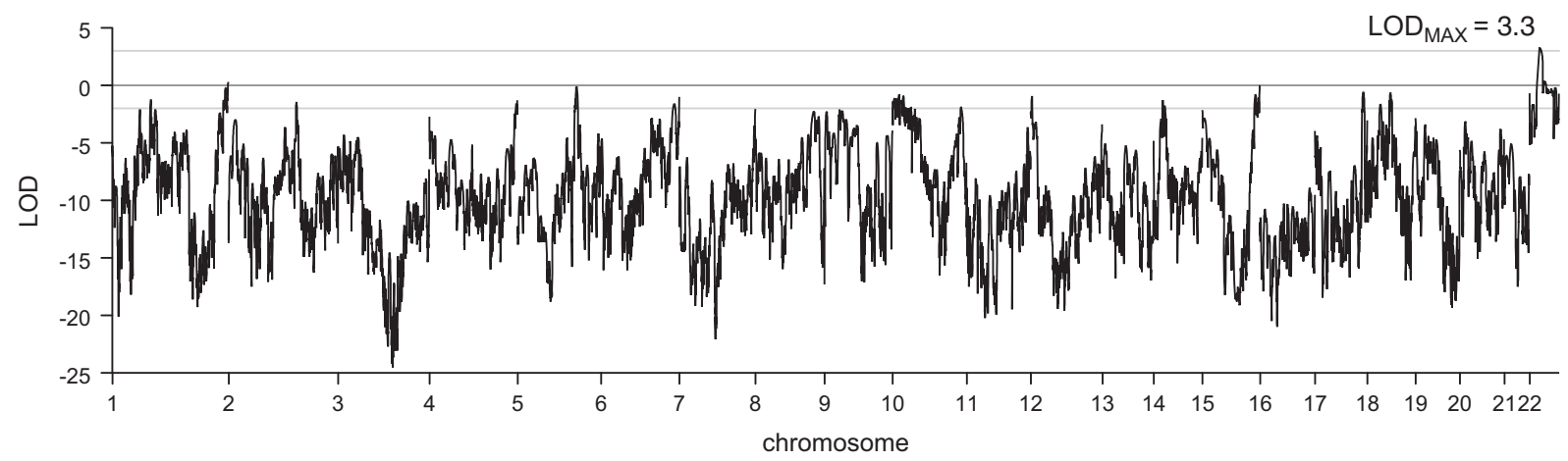

b

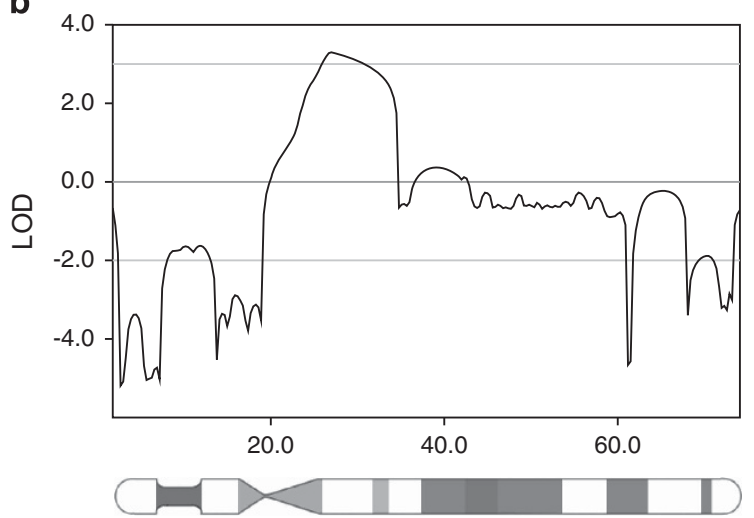

d

\begin{tabular}{lllll} 
symbol & gene & chr & start & end \\
MYO18B & myosin XVIIIB & 22 & 26138117 & 26453345 \\
SEZ6L & seizure related 6 homolog (mouse)-like & 22 & 26565440 & 26779563 \\
ASPHD2 & aspartate beta-hydroxylase domain containing 2 & 22 & 26825280 & 26840978 \\
HPS4 & Hermansky-Pudlak syndrome 4 & 22 & 26846848 & 26879829 \\
SRRD & SRR1 domain containing & 22 & 26879850 & 26887904 \\
TFIP11 & tuftelin interacting protein 11 & 22 & 26887893 & 26908462 \\
TPST2 & tyrosylprotein sulfotransferase 2 & 22 & 26921714 & 26986089 \\
\hline CRYBB1 & crystallin, beta B1 & 22 & 26995362 & 27013991 \\
CRYBA4 & crystallin, beta A4 & 22 & 27017928 & 27026636 \\
\hline MN1 & meningioma (disrupted in balanced translocation) 1 & 22 & 28144265 & 28197486 \\
PITPNB & phosphatidylinositol transfer protein, beta & 22 & 28247657 & 28315255 \\
TTC28 & tetratricopeptide repeat domain 28 & 22 & 28374002 & 29075853 \\
CHEK2 & checkpoint kinase 2 & 22 & 29083731 & 29137822 \\
HSCB & HscB mitochondrial iron-sulfur cluster co-chaperone & 22 & 29138008 & 29153506 \\
CCDC117 & coiled-coil domain containing 117 & 22 & 29168662 & 29185283 \\
XBP1 & X-box binding protein 1 & 22 & 29190548 & 29196560 \\
ZNRF3 & zinc and ring finger 3 & 22 & 29279755 & 29453476
\end{tabular}

Chromosome $22(\mathrm{cM})$

C

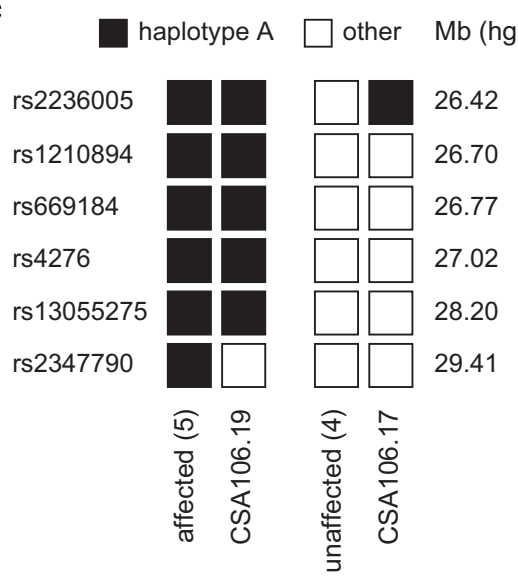

e

\begin{tabular}{|c|c|c|}
\hline total variants & 45 & \\
\hline shared heterozygous & 56 & \\
\hline absent from unaffected & 5 & \\
\hline nonsyn/stop/frameshift/splice & 3 & \\
\hline hg19 chr22:2g.6422980-29414001 & 2 & $\begin{array}{l}\text { SEZ6L p.(Trp185Leu) (ExAC MAF:0.04381) } \\
\text { HPS4 p.(Ile84Val) (ExAC MAF:0.004654) }\end{array}$ \\
\hline
\end{tabular}

Figure 2 Linkage of the cataract phenotype to proximal chromosome 22q. (a) Genome-wide LOD scores under a fully penetrant dominant inheritance model. (b) LOD scores across chromosome 22. (c) Haplotype analysis and critical recombinants across the interval. (d) List of protein-coding genes within the defined linkage interval, with previously known congenital cataract genes highlighted. Start and end coordinates refer to the hg19 reference sequence. (e) Filtering strategy to identify shared heterozygous coding variants within linkage interval.

(Figure $4 \mathrm{~b}$, underlined). A predicted consequence of this proximal breakpoint was the creation of a hybrid CRYBB1 gene. This gene encoded the expected CRYBB1 amino acid sequence up to and including amino acid 205 (a glutamine residue). Beyond this point (corresponding to the proximal breakpoint) the final 47 C-terminal amino acids, including the majority of the fourth and final 'Greek key' crystallin domain, are predicted to be replaced by 18 unrelated amino acids encoded by intergenic sequence (Figure $4 \mathrm{~b}$ and c). If transcribed, this product would also be predicted to avoid nonsense-mediated decay, as the premature termination signal occurs in the final exon.

\section{DISCUSSION}

Here we describe an autosomal dominant congenital cataract pedigree with unambiguous linkage to chromosome 22. Despite the absence of a candidate single nucleotide variant in the coding regions of genes within the linked interval, we identified a partial direct tandem duplication of the CRYBB1-CRYBA4-MIAT locus. Although we have not definitively ascribed pathogenicity to this duplication, there are precedents for both CRYBB1 and CRYBA4 causing dominant congenital cataract.

Variants in CRYBA4, for example, have been described in autosomal dominant congenital cataract. ${ }^{7,13}$ Both reports describe missense 
a

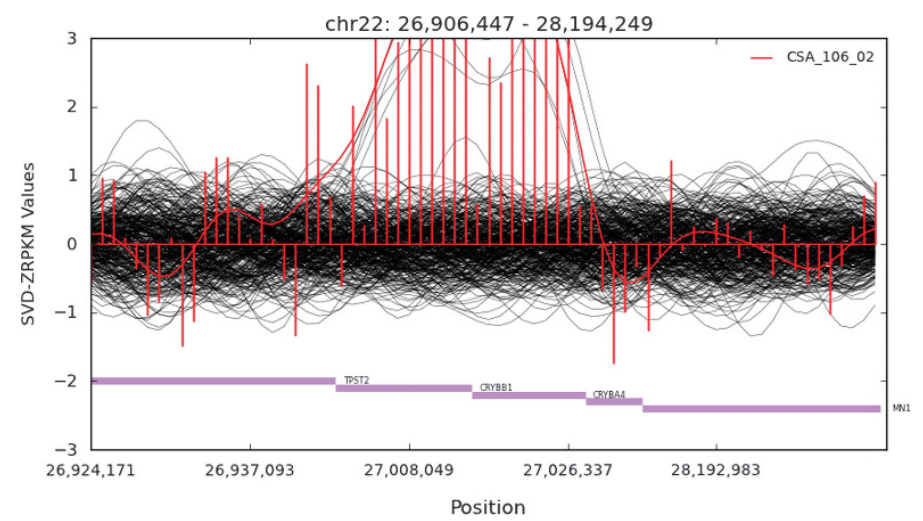

b

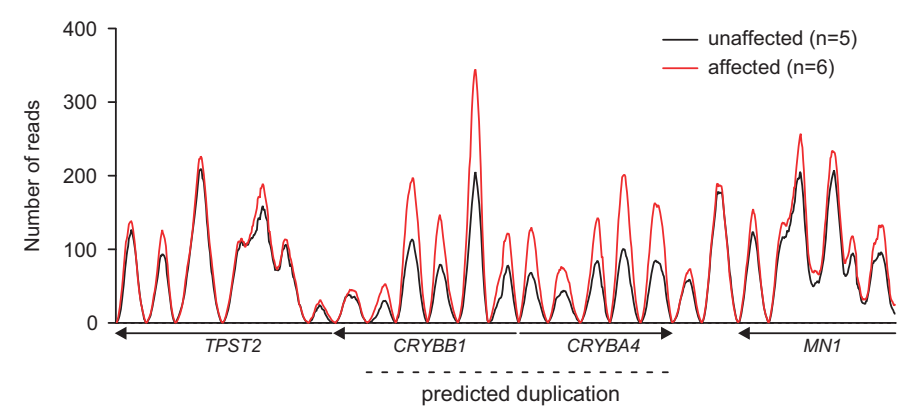

d

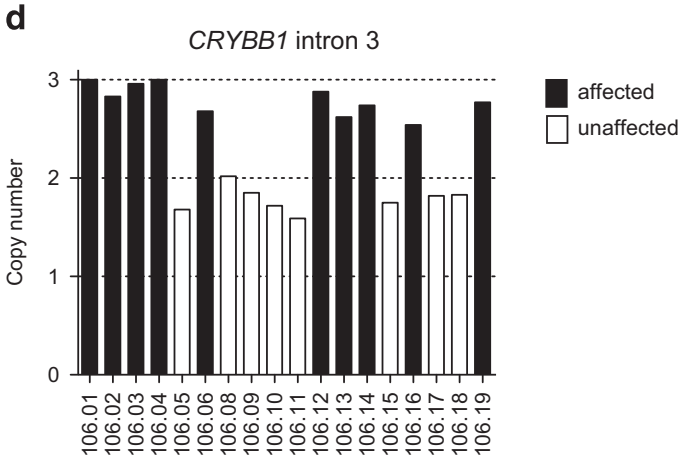

CRYBB1 exon 6

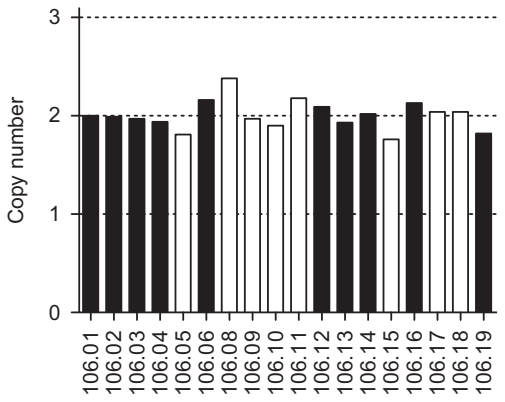

C

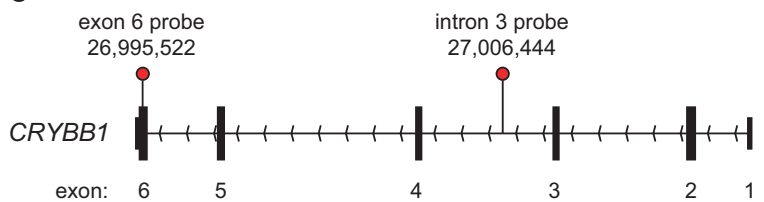

f

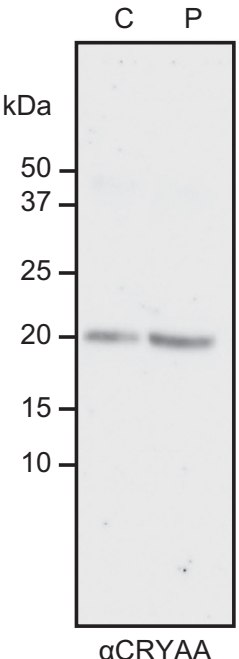

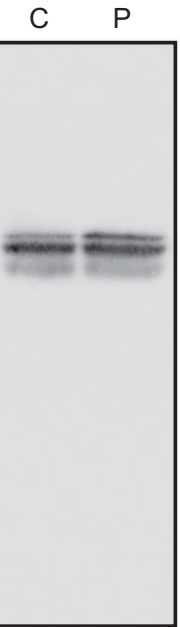

aCRYBB1
C $P$

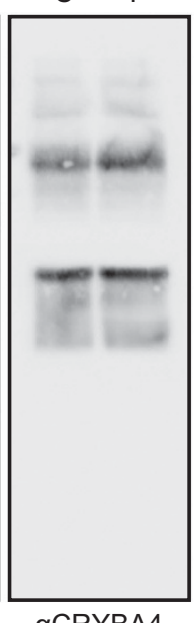

aCRYBA4 e

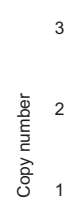

CRYBB1 intron 3

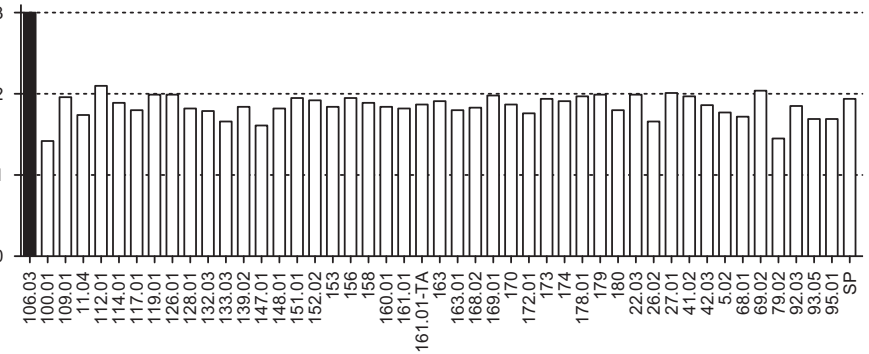

Figure 3 Partial duplication of the CRYBB1-CRYBA4 locus. (a) CoNIFER output indicating a copy number gain within the captured portion of the linkage interval. (b) Average read depth of affected (red) and unaffected (black) family members across the same interval shown in (a). Each peak represents a region covered by exome capture and sequencing, typically an exon. All five coding exons of both CRYBB1 and CRYBA4 were covered, with an additional region captured and sequenced for CRYBB1. The final peak at the CRYBB1 locus (ie, furthest to left) represents the sixth and final exon. (c) Exon structure of CRYBB1 and location of qPCR probes used for copy number variant analysis. (d) qPCR validation of a duplicated (intron 3) and non-duplicated (exon 6) region of $C R Y B B 1$. Bars indicate copy number $(C N)$ normalised to an internal control probe. (e) CRYBB1 duplication screening in unsolved congenital cataract cases. (f) Crystallin protein content in cataractous lens extracts. Control [c] and patient $[\mathrm{P}]$ lens extracts were subjected to Western blotting with the indicated antibodies. CRYAA served as a loading control, with the same blot probed sequentially with all three antibodies. 
variants (c.225G $>\mathrm{T} \quad$ (p.(Gly64Trp)), c.242T $>\mathrm{C} \quad$ (p.(Leu69Pro)), c.317T $>$ C (p.(Phe94Ser))) which presumably promote cataract formation by creating a less soluble protein. Yet the CRYBA4 duplication described here covered the complete gene, did not contain any missense variants, and did not lead to any obvious change in protein expression.

a

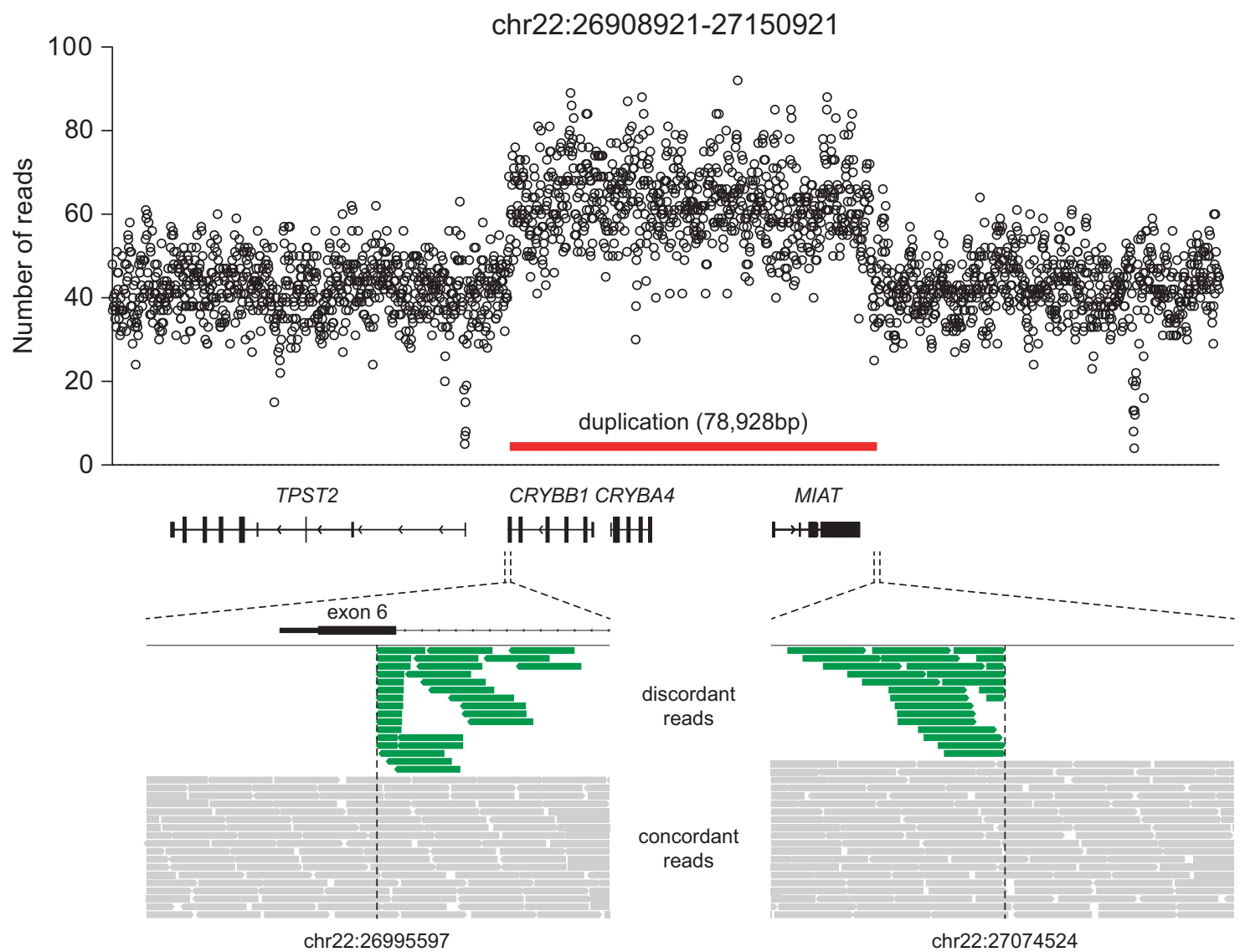

b

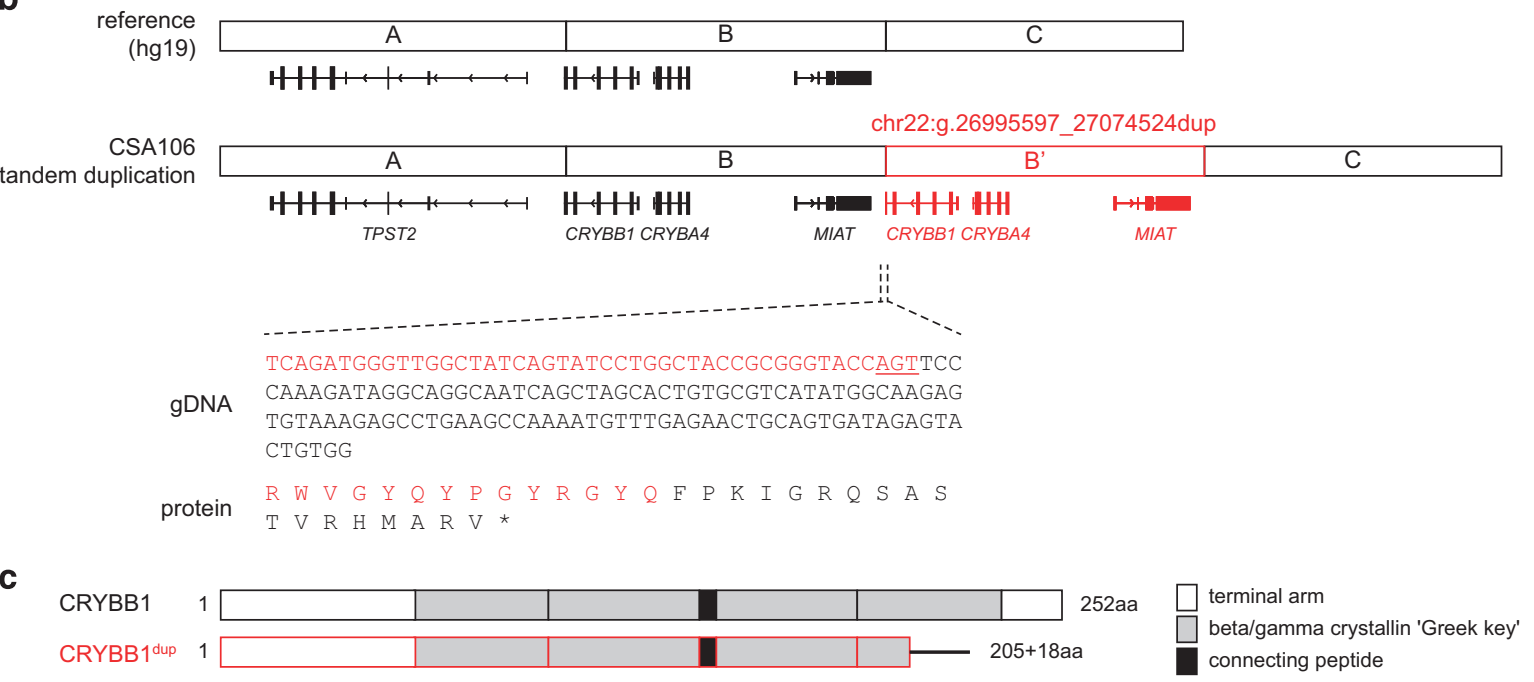

Figure $4 \mathrm{~A}$ direct tandem duplication at the CRYBB1-CRYBA4 locus resulting in partial duplication of CRYBB1. (a) Whole-genome sequencing depth across the CRYBB1-CRYBA4 locus of an affected family member (CSA106.19). Relative location of local transcripts, concordant (grey) and discordant (green) paired reads, and duplication boundaries are indicated below. (b) Depiction of the CSA106 tandem duplication, showing the sequence of a 150 bp split read spanning the duplication breakpoint (gDNA) and its predicted translation product (protein). Red gDNA sequence aligns to exon 6 of the duplicated CRYBB1 gene, with black sequence aligning to a region distal to the long non-coding RNA, MIAT. A 3 bp region of microhomology is underlined. Predicted translation of the mate pair sequence reveals 14 amino acids from the C-terminus of CRYBB1 (red), followed by an 18 amino acid read-through product and premature termination codon (black). (c) Domain structure of full-length CRYBB1 protein, and hybrid CRYBB1 protein produced as a consequence of the CSA106 tandem duplication (CRYBB1 ${ }^{\text {dup}}$ ). 
On the other hand, CRYBB1 variants appear to have two distinct pathways to cataractogenesis. The recessive CRYBB1 variants c.169delG (p.(Gly57Glyfs $\left.{ }^{\star} 107\right)$ ) or c.2T $>$ A (p.(Met1Lys)) are not expected to be expressed at all, and presumably cause cataracts by completely removing an important structural component. ${ }^{9,14}$ Conversely the dominant alleles such as c.658G $>\mathrm{T}\left(\mathrm{p} .\left(\right.\right.$ Gly220 $\left.20^{\star}\right){ }^{8}$ c. $737 \mathrm{C}>\mathrm{T}\left(\mathrm{p} .\left(\mathrm{G} \ln 223^{\star}\right)\right)^{15}$ and c. $827 \mathrm{~T}>\mathrm{C}\left(\mathrm{p} .\left({ }^{*} 253 \mathrm{Arg}\right)\right)^{16}$ are predicted to cause cataract by disrupting the coding sequence of the final exon (exon 6), and creating a protein with reduced solubility. The hybrid CRYBB1 gene created as a consequence of the duplication described here also disrupts the coding sequence of exon 6, and is associated with an autosomal dominant pattern of inheritance. We therefore consider it most likely that the CRYBB1 duplication product is the disease-causing agent, despite the fact that we did not detect an appropriately-sized band by western blotting.

The absence of an additional CRYBB1 band on western blot may still be consistent with a gain-of-function mechanism: for example, the duplicated CRYBB1 product may have only been transiently expressed, or has a shorter half-life than full-length CRYBB1. Conversely it may not have been synthesised at all, and played no role in cataractogenesis in this family. Given this possibility, we have not excluded the possibility that CRYBA4 triplosensitivity was responsible for the cataractogenesis, which could conceivably alter the stoichiometry of crystallin subunits at a critical stage of lens development. It is also possible that local transcription is altered in the context of a tandem duplication, again raising implications for crystallin stoichiometry. Duplication of the MIAT long noncoding RNA seems the least likely explanation for cataractogenesis in this family, given the large body of evidence for CRYBB1 and CRYBA4 in congenital cataract.

More broadly, copy number variation is largely overlooked in many exome and genome sequencing studies, perhaps due in part to the limited predicted contribution of CNVs to common disease. ${ }^{17}$ In cases where CNVs do play a role it is almost always deletions that are responsible, either in trans with a second deleterious allele, or by causing haploinsufficiency on their own. Increases in copy number are far less common in a disease setting, and when they do occur, they commonly involve complete genes. Ocular disease is no exception, with duplication or triplication of TBK1 in normal tension glaucoma being one example, ${ }^{18,19}$ and a complex NHS triplication in the congenital cataract-associated Nance-Horan syndrome being another. ${ }^{20}$ TBK1 $\mathrm{CNVs}$ associated with glaucoma cover the entire locus, so a mechanistic explanation has not been immediately obvious. In the case of the Nance-Horan syndrome triplication, disruption of NHS transcription is thought to explain the phenotype, which is consistent with the lossof-function mechanism of other NHS variants..$^{20}$ In a third example, both deletions and duplications of the same gene (FOXC1) have been associated with anterior segment dysgenesis. ${ }^{21}$

Other diseases can be caused by partial gene duplication, ${ }^{22}$ including $\sim 7 \%$ of cases of the X-linked Duchenne and Becker muscular dystrophies (DMD/BMD). ${ }^{23}$ In the case of DMD these variants invariably cause a frameshift, whereas in BMD the reading frame is maintained. ${ }^{22}$ In both cases the predicted result is a loss or reduction in protein function.

Duplication has been integral to the diversification of the crystallin gene family. In the family presented here, we show that crystallin duplication can also be associated with congenital cataract.
This represents a previously undescribed genetic mechanism for the development of isolated congenital cataract, with implications for other inherited diseases that appear refractory to exome or genome sequencing.

\section{CONFLICT OF INTEREST}

The authors declare no conflict of interest.

\section{ACKNOWLEDGEMENTS}

We thank Angela Chappell for cataract photography. Supported by the National Health and Medical Research Council (NHMRC) grant 1023911. KPB is funded by an NHMRC Senior Research Fellowship, and JEC by an NHMRC Practitioner Fellowship.

1 Bourne RRA, Stevens GA, White RA et al: Causes of vision loss worldwide, 1990-2010: a systematic analysis. Lancet Glob Health 2013; 1: e339-e349.

2 Haargaard B, Wohlfahrt J, Fledelius HC, Rosenberg T, Melbye M: Incidence and cumulative risk of childhood cataract in a cohort of 2.6 million Danish children. Invest Ophthalmol Vis Sci 2004; 45: 1316-1320.

3 Haargaard B, Wohlfahrt J, Fledelius HC, Rosenberg T, Melbye M: A nationwide Danish study of 1027 cases of congenital/infantile cataracts: etiological and clinical classifications. Ophthalmology 2004; 111: 2292-2298.

4 Shiels A, Bennett TM, Hejtmancik JF: Cat-Map: putting cataract on the map. Mol Vis 2010; 16: 2007-2015.

5 Shiels A, Hejtmancik JF: Genetics of human cataract. Clin Genet 2013; 84: 120-127. 6 Wistow G: The human crystallin gene families. Hum Genomics 2012; 6: 26.

7 Billingsley G, Santhiya ST, Paterson AD et al: CRYBA4, a novel human cataract gene, is also involved in microphthalmia. Am J Hum Genet 2006; 79: 702-709.

8 Mackay DS, Boskovska OB, Knopf HLS, Lampi KJ, Shiels A: A nonsense mutation in CRYBB1 associated with autosomal dominant cataract linked to human chromosome 22q. Am J Hum Genet 2002; 71: 1216-1221.

9 Cohen D, Bar-Yosef U, Levy J et al: Homozygous CRYBB1 deletion mutation underlies autosomal recessive congenital cataract. Invest Ophthalmol Vis Sci 2007; 48: 2208-2213.

10 Smith KR, Bromhead CJ, Hildebrand MS et al: Reducing the exome search space for mendelian diseases using genetic linkage analysis of exome genotypes. Genome Biol 2011; 12: R85.

11 Buhr F, Jha S, Thommen M et al: Synonymous Codons Direct Cotranslational Folding toward Different Protein Conformations. Mol Cell 2016; 61: 341-351.

12 Newman S, Hermetz KE, Weckselblatt B, Rudd MK: Next-generation sequencing of duplication CNVs reveals that most are tandem and some create fusion genes at breakpoints. Am J Hum Genet 2015; 96: 208-220.

13 Zhou G, Zhou N, Hu S, Zhao L, Zhang C, Qi Y: A missense mutation in CRYBA4 associated with congenital cataract and microcornea. Mol Vis 2010; 16: 1019-1024.

14 Meyer E, Rahman F, Owens J et al: Initiation codon mutation in betaB1-crystallin (CRYBB1) associated with autosomal recessive nuclear pulverulent cataract. Mol Vis 2009; 15: 1014-1019.

15 Yang J, Zhu Y, Gu F et al: A novel nonsense mutation in CRYBB1 associated with autosomal dominant congenital cataract. Mol Vis 2008; 14: 727-731.

16 Willoughby CE, Shafiq A, Ferrini W et al: CRYBB1 mutation associated with congenital cataract and microcornea. Mol Vis 2005; 11: 587-593.

17 Conrad DF, Pinto D, Redon R et al: Origins and functional impact of copy number variation in the human genome. Nature 2010; 464: 704-712.

18 Awadalla MS, Fingert JH, Roos BE et al: Copy number variations of TBK1 in Australian patients with primary open-angle glaucoma. Am J Ophthalmol 2015; 159: 124-130.e1.

19 Fingert JH, Robin AL, Stone JL et al: Copy number variations on chromosome 12q14 in patients with normal tension glaucoma. Hum Mol Genet 2011; 20: 2482-2494.

20 Coccia M, Brooks SP, Webb TR et al: X-linked cataract and Nance-Horan syndrome are allelic disorders. Hum Mol Genet 2009; 18: 2643-2655.

21 Nishimura DY, Searby CC, Alward WL et al: A spectrum of FOXC1 mutations suggests gene dosage as a mechanism for developmental defects of the anterior chamber of the eye. Am J Hum Genet 2001; 68: 364-372.

$22 \mathrm{Hu} \mathrm{X}$, Worton RG: Partial gene duplication as a cause of human disease. Hum Mutat 1992; $1: 3-12$

23 White SJ, Aartsma-Rus A, Flanigan KM et al: Duplications in the DMD gene. Hum Mutat 2006; 27: 938-945.

Supplementary Information accompanies this paper on European Journal of Human Genetics website (http://www.nature.com/ejhg) 